\title{
Estimating demographic parameters for capture-recapture data in the presence of multiple mark types
}

\author{
Sophie Smout • Ruth King • Patrick Pomeroy
}

Received: 27 May 2009 / Revised: 3 December 2009

(C) Springer Science+Business Media, LLC 2010

\begin{abstract}
In mark-recapture studies, various techniques can be used to uniquely identify individual animals, such as ringing, tagging or photo-identification using natural markings. In some long-term studies more than one type of marking procedure may be implemented during the study period. In these circumstances, ignoring the different mark types can produce biased survival estimates since the assumption that the different mark types are equally catchable (homogeneous capture probability across mark types) may be incorrect. We implement an integrated approach where we simultaneously analyse data obtained using three different marking techniques, assuming that animals can be cross-classified across the different mark types. We discriminate between competing models using the AIC statistic. This technique also allows us to estimate both relative mark-loss probabilities and relative recapture efficiency rates for the different marking methods. We initially perform a simulation study to explore the different biases that can be introduced if we assume a homogeneous recapture probability over mark type, before applying the method to a real dataset. We make use of data obtained from an intensive long-term observational study of UK female grey seals (Halichoerus grypus) at a single breeding colony, where three different methods are used to identify individuals within a single study: branding, tagging and photo-identification based on seal coat pattern or pelage.
\end{abstract}

\footnotetext{
S. Smout $(\varangle)$

Centre for Research into Ecological and Environmental Modelling, University of St. Andrews, Buchanan Gardens, St. Andrews, Fife KY16 9LZ, Scotland, UK

e-mail: scs10@st-andrews.ac.uk; scs10@st-and.ac.uk

R. King

School of Mathematics and Statistics, University of St. Andrews, Fife, Scotland, UK

P. Pomeroy

Sea Mammal Research Unit, University of St. Andrews, Fife, Scotland, UK
}

Published online: 14 March 2010 
Keywords Mark-recapture · Mark-loss · Halichoerus grypus · Multiple mark types · Integrated analysis

\section{Introduction}

In mark-recapture studies of open biological populations a number of simplifying assumptions are often made. These include no loss of marks from individuals; all identifying marks are unique; marks are never mis-read so that animals are always correctly identified; and the sample is representative of the population. Unfortunately, some (or all) of these assumptions are not always valid and these aspects are often of particular interest. For example, in the case where individual marks may be lost, the probability of losing a mark is often of interest in itself. Alternatively, recapture probabilities are typically assumed to be homogeneous over individuals, however, this may not generally be the case, particularly where different marking methods are applied to different individuals. We consider the case where there are multiple methods for marking individuals. In some cases, individuals may carry more than one mark type; when this is the case, we assume that individuals can be cross classified across marks. For the particular application considered, these are brands, tags and photo-identification. Ignoring the different mark types and assuming that recapture probabilities are homogeneous over mark type can potentially lead to biased results. We develop an integrated approach where we simultaneously analyse all available data, modelling explicitly the effect of mark type on the recapture probability of an individual. In particular, we allow the recapture probability to vary with respect to the mark type applied to the individual, but assume that the recapture probability is homogeneous over individuals with a given mark type. We note that this approach is similar to that of Lebreton et al. (1992) who adopt a stratified CJS-type model where groups are distinguished by mark type; and Pledger et al. (2003) who propose mixture models to allow for heterogeneity amongst different individuals for both recapture and survival probabilities but where group membership is not known for individual animals. Within our approach the mixture component that each individual belongs to is known, corresponding to the type of mark applied to the animal for unique identifiability. We consider reparameterisations of the model (and additional sub-models) to represent interpretable biological parameters of interest, including relative mark recapture (efficiency) rates and mark-loss probabilities.

The effect of mark-loss on the estimation of survival probabilities is well-known. Estimates of survival probabilities are negatively biased in the presence of unaccounted-for mark-loss. In particular the survival estimates obtained are confounded with mark-loss (and permanent migration from the study site). Thus, without additional information only apparent survival probabilities can be estimated from standard mark-recapture data. In other words we refer to the "death" of an individual to mean that the animal ceases to be within the study as a result of actual death, loss of the unique identification mark, or permanent migration from the colony. To remove the confounding of mark-loss with survival (including permanent migration) a common approach is to introduce "double-tagging". See, for example, Barrowman and Myers (1996), Kremers (1988), Conn et al. (2004) and Cowen et al. (2009) for a variety of 
different applications. Within double-tagging experiments the tag-loss rate is essentially estimable by the observation of single tag-losses from double-tagged individuals within the data. However, typically a number of assumptions are made such as equal observability of single and double marked individuals and independent tag-loss rates (Testa and Rothery 1992). Methodology for addressing the issue of non-independent tag loss has been investigated by McMahon and White (2009), Bradshaw et al. (2000) and Diefenbach and Alt (1998). An alternative scenario for collecting data to estimate tag-loss is to use different mark types. For example, within bird studies, Kremers (1988) used two different types of rings placed on the same individual-non-permanent colour-coded rings that could be resighted and permanent metal rings that could only be identified upon recovery (following the death of an individual). Reynolds et al. (2009) consider a similar approach, using the different types of rings to separately estimate both tag-loss rates and permanent emigration. We consider a different situation where we only observe live recaptures. In addition, the different mark types are placed on distinct individuals, and three different types of markings are used on a single population (permanent brands, tags and natural pelage markings). We are then able to provide estimates of mark-loss probabilities for tag and pelage individuals by essentially comparing the capture histories with those for the branded seals where we assume there is no brand-loss (and allowing for different recapture probabilities for the different mark types).

In Sect. 2 we describe in detail the data collected on the UK grey seal (Halichoerus grypus) breeding colony of interest. We then develop the models that we fit to the data in Sect. 3, permitting the estimation of mark-loss probabilities and recapture efficiency rates. In Sect. 4 we perform a simulation study to assess the bias that can be introduced by ignoring the issue of mark-loss and differing recapture probabilities dependent on mark type. We return to the seal data in Sect. 5, fitting and comparing a number of different models before discussing the results obtained in terms of the biological interpretation. Finally we conclude with a discussion in Sect. 6.

\section{Data}

The breeding colony of grey seals on North Rona (NR), Scotland $\left(59^{\circ} 06^{\prime} \mathrm{N}, 05^{\circ} 50^{\prime} \mathrm{W}\right)$ has been the focus of population studies since the 1960s (Boyd and Laws 1962). Individual female grey seals haul out to give birth between September and November, when the capture-recapture data are collected. Each female produces a single pup which she attends and suckles over a period of approximately 18 days. Mothers remain ashore for an average of 20 days fasting, during which time they give birth, suckle their pups, mate and return to the sea (Pomeroy et al. 1999). On NR it is uncommon for mothers to return to the sea during the breeding period. Pups are weaned abruptly when mothers leave the colony. Females show considerable fidelity to their natal colony and often return to breed at the same location within the colony over many years (Pomeroy et al. 2000). However, it is feasible that an individual may be absent from the colony in a given year. We assume that any absences from a colony can be modelled by random temporary emigration. For an individual who is absent in a given year, its breeding status is unknown: they may breed elsewhere, or they may skip breeding. Finally, we 
assume that there is no mortality during the breeding season, and that individuals that die cannot be recovered within the study.

Capture-recapture data are collected each year researchers are present on NR. We consider data for adult females from 1985-2006 and note that no data were collected in 1990, 1991 or 1992 . The time series of data spans over 30 years, but changes in the scientific focus and development of procedures during the work have meant that a number of different marking methods have been used to identify individual seals. These include branding, flipper-tags, and photo-identification, which makes use of the natural variation in coat patterns (pelage) between individual seals (Hiby and Lovell 1990; Vincent et al. 2001). Individuals were identified via photographs of pelage patterns taken during the field season, often using telescopic equipment so that records could be collected from some distance away (approximately $200 \mathrm{~m}$ ), thus minimising disturbance to the study animals. Each branded animal was marked with a unique letter/number cipher on both flanks and a recapture was recorded when these could be read clearly. Flipper tags were placed on the hind flippers of the seals, and tag re-sightings were recorded only when an observer was able to read the unique code on a tag. Due to the careful collection of data on observed individuals, we assume that all individuals are correctly identified for each mark type.

Sightings of marked seals were recorded each year. An example of a typical capture history would be of the form:

\section{brand $011111101 \ldots 0$.}

The first column denotes the mark type associated with the individual seal (a brand in this case) and the series of 0 s and 1 s the usual capture-recapture record denoting that an individual was either observed (1) or not observed (0) each year within the study. For the dataset considered here, a total of 106 branded seals (78 of these branded in the two years 1985 and 1987); 29 tagged seals (marked as adults from 1985 onwards) and 341 pelaged seals (photo-identified between 1996-present) are uniquely identified. The number of different marks applied to the seals in each year of the study is provided in Table 1.

We note that some animals in the study carry more than one mark type for a number of years in the study. However, for simplicity within this analysis, we use the following protocol for assigning individuals to a single mark type at any point within the study. From practical biological fieldwork, brands are most easily observed as they are very distinct and can be observed from a distance. Animals identified via their pelage can again be observed from a distance, but photo-identification is needed. Tags are typically the hardest marks to observe, as they need to be observed at close-hand and can often be obscured. Thus, we assume that brands "dominate" pelage which in turn "dominate" tags, where "dominate" here refers to the method most likely to result in an individual being observed if that individual has more than one mark type at any given time. Thus, using this reasoning, we assume "branded" animals correspond to individuals with a brand (with or without any other mark type); "pelaged" animals are identified via their pelage (with or without a tag) and "tagged" animals are individuals with only a tag (with no other mark type). Note that an individual can effectively be removed from the study (or lost on capture) and subsequently reintroduced as a distinct 
Table 1 The number of mark types applied in each year throughout the study period

\begin{tabular}{|c|c|c|c|}
\hline \multirow[t]{2}{*}{ Year } & \multicolumn{3}{|c|}{ Mark type } \\
\hline & Brand & Tag & Pelage \\
\hline 1985 & 63 & 5 & 0 \\
\hline 1986 & 1 & 4 & 0 \\
\hline 1987 & 15 & 4 & 0 \\
\hline 1988 & 6 & 0 & 0 \\
\hline 1989 & 0 & 2 & 0 \\
\hline 1990 & 0 & 0 & 0 \\
\hline 1991 & 0 & 0 & 0 \\
\hline 1992 & 0 & 0 & 0 \\
\hline 1993 & 0 & 1 & 0 \\
\hline 1994 & 2 & 1 & 0 \\
\hline 1995 & 3 & 0 & 0 \\
\hline 1996 & 4 & 1 & 4 \\
\hline 1997 & 0 & 2 & 13 \\
\hline 1998 & 8 & 1 & 47 \\
\hline 1999 & 3 & 1 & 53 \\
\hline 2000 & 1 & 3 & 41 \\
\hline 2001 & 0 & 3 & 4 \\
\hline 2002 & 0 & 1 & 125 \\
\hline 2003 & 0 & 0 & 42 \\
\hline 2004 & 0 & 0 & 4 \\
\hline 2005 & 0 & 0 & 5 \\
\hline
\end{tabular}

individual if a "dominating" mark is applied at some point within its capture history. We treat the individual as being removed from the study at the time the dominating mark is added and a new history is created corresponding to the capture history of the individual following the application of the new dominating mark. (We discuss this issue further in Sect. 6).

\section{Method}

Within the analysis of open capture-recapture data, where we condition on the initial capture of an individual, the parameters of primary interest are typically the survival probabilities, with the recapture probabilities essentially nuisance parameters. However, as is often the case with nuisance parameters, reliably estimating the recapture probabilities is essential in obtaining reliable estimates for the survival probabilities (Seber 1982). Thus, the underlying model specified on not only the survival probabilities, but also the recapture probabilities is important in any statistical analysis. Within our capture-recapture study, the different mark types may have different 
associated "catchabilities". We can account for this capture heterogeneity by considering distinct recapture probabilities for the different mark types. The relative values of these recapture probabilities may also be of interest as they enable us to compare the efficiency with which seals carrying the three types of mark can be re-sighted by observers. In addition, relative mark-loss probabilities can be calculated, which may be of particular importance in obtaining unbiased estimates of the durability of the marks, essential for planning future capture-recapture studies.

We consider an analysis of all the available capture-recapture data, taking into account the different marking types within a single integrated framework. We can then use the standard Cormack-Jolly-Seber (CJS) model (Cormack 1964; Seber 1965; Jolly 1965), essentially taking the mark type as a discrete covariate for the demographic parameters. For time $t=1, \ldots, T-1$, we define the following parameters for individuals with mark type $m \in\{$ brand, tag, pelage $\}$ :

$$
\begin{aligned}
& \phi_{m}(t)= \mathbb{P}(\text { an individual identified via mark type } m \text { survives until } \\
&\text { time } t+1 \mid \text { alive at time } t) \\
& p_{m}(t+1)= \mathbb{P}(\text { an individual identified via mark type } m \text { is observed at } \\
&\text { time } t+1 \mid \text { individual alive at time } t+1) .
\end{aligned}
$$

For notational convenience, we set $\boldsymbol{\phi}=\left\{\phi_{m}(t): t=1, \ldots, T-1 ; m \in\right.$ $\{$ brand,tag, pelage $\}, \boldsymbol{p}=\left\{p_{m}(t): t=2, \ldots, T ; m \in\{\right.$ brand, tag, pelage $\}$ and $\boldsymbol{\theta}=\{\boldsymbol{p}, \boldsymbol{\phi}\}$. We note that the survival probability $\phi_{m}(t)$ is essentially an apparent survival probability, with death confounded with mark-loss and permanent migration. The likelihood is then proportional to the product over each observed individual of the probability of their corresponding capture history (and a relatively simple function of the survival and recapture probabilities).

This "global" model assumes that the survival and recapture probabilities are fully time and mark dependent. This model is parameter redundant, since $\phi_{m}(T-1)$ and $p_{m}(T)$ are confounded for each $m \in\{$ brand, tag, pelage $\}$ (see for example Catchpole et al. 2002). Reparameterising the model and considering restrictions specified on the parameters provides us with additional insight into the observation process for the population under study. In addition, placing the restrictions on the parameters such as a constant survival probability or recapture probability can remove the parameter redundancy. We reparameterise the model in such a way that we are able to estimate mark loss probabilities, and by comparing the capture probabilities for the different mark types, obtain relative capture efficiency rates for the different marking methods. We discuss each of these in turn.

\subsection{Estimating mark-loss rates}

We assume that marking does not affect the general behaviour or survival of the individuals following the application of the mark, and thus we expect actual mortality and permanent migration probabilities of individuals to be equal across mark types. It is this assumption that allows us to estimate relative mark-loss rates, essentially 
by comparing the apparent survival probabilities between the different mark-types. However, here we also assume that brands are permanent and hence cannot be "lost" (a reasonable assumption in this case). Thus we let $\phi_{\text {brand }}(t)=\phi(t)$, where $\phi(t)$ corresponds to the probability that an individual survives from time $t$ to $t+1$ (given they are alive at time $t$ ) and returns to the study area. In other words, we can remove the mark-loss component of the apparent survival probability since we assume that there is no brand-loss. For ease of reference we refer to $\phi(t)$ simply as the survival probability. Assuming a time-dependent mark-loss probability, we can reparameterise the model by specifying:

$$
\begin{aligned}
\phi_{\text {tag }}(t) & =\left(1-a_{\text {tag }}(t)\right) \phi(t) ; \\
\phi_{\text {pelage }}(t) & =\left(1-a_{\text {pelage }}(t)\right) \phi(t) .
\end{aligned}
$$

Here $a_{\text {tag }}(t)$ and $a_{\text {pelage }}(t)$ denote the corresponding probabilities that an individual loses its identifying mark, (tag or pelage), in the interval $[t, t+1$ ), so that $a_{\text {tag }}(t), a_{\text {pelage }}(t) \in[0,1]$. We can regard this model as a "restricted" model, in that the survival probabilities of tagged and pelaged animals can be at most equal to the survival probability, $\phi(t)$. Thus, this model is not necessarily equivalent to the (unrestricted) "global" CJS model described above, due to the restrictions placed on the parameter $a_{\text {tag }}(t)$ and $a_{\text {pelage }}(t)$. In general, if there is possible brand-loss, the interpretation of $a_{\text {tag }}(t)$ and $a_{\text {pelage }}(t)$ would be of the relative tag-loss or pelage-loss, and would not necessarily need to be restricted to be $\leq 1$ (if for example, brand-loss was greater in one year than tag-loss). Finally, we note that we can extend these models to allow for tag-age dependent mark-loss rates. Such models may incorporate, for example, the fact that older tags may be more readily lost as the tags degrade with time. We would then have the analogous $a_{\text {tag }}(t, l)$ term, where $l$ corresponds to the age of the tag [see for example Cowen and Schwarz (2006) for further discussion of similar tag-loss models]. However, we do not consider this case further, as we do not have sufficient data in our example to estimate age-dependent tag-loss.

The pelage of female seals does not generally change significantly once the first adult coat is grown (Vincent et al. 2001), which would mean that there is no pelageloss (i.e. $a_{\text {pelage }}(t)=0$, for $t=1, \ldots, T-1$ ). However, to allow for the possibility of a change in the pelage we retain the possibility of pelage loss in the most general model, though we might expect this parameter to be small in value. A sub-model of particular interest in the estimation of mark-loss is to consider mark-loss as a constant, time-invariant, quantity. For example, this sub-model is obtained by setting

$$
a_{t a g}(t)=a_{t a g}
$$

for all $t=1, \ldots, T-1$.

We note that there is significant overlap in the presence records (i.e. observed 1s) in the capture-recapture histories of the seals with different mark types (although the number of tagged animals is small in our case). This is generally necessary in order to provide reliable estimates of the survival and mark-loss probabilities.

Finally, we comment on the parallel of this approach with that applied in the presence of reward tags (Pollock et al. 2001). This approach involves two types of tags- 
reward tags and standard tags. Large rewards are provided for the return of tags, so that it can be assumed that the return probability of a reward tag is $100 \%$. The relative return rate of standard tags, assuming no tag-loss, can then be estimated by comparing the relative standard tag reporting rate to high rewards tags for tag recovery data i.e. observed deaths. In our case we observe live resightings with the relative difference in the survival probabilities for the different mark types used to estimate mark-loss rates, assuming the brand retention is $100 \%$ (i.e. there is no brand loss).

\subsection{Estimating recapture efficiency}

In this study, we expect the recapture probabilities to vary with respect to the mark type. Some mark types are more easily seen and read than others. For example, brands can be seen through binoculars from a distance while tags must be observed at closer range, and are sometimes obscured by the folding of the seal's flippers or the position of the seal. This variability with respect to resighting probability is once more analogous to the ideas associated with reward tagging methods, but in relation to recapture probabilities rather than recovery probabilities. Note that within the method of reward tagging, the use of reward tags may also result in a change in the reporting probabilities of standard tags. This complication does not occur in our case with the use of multiple marks. Conversely, we are only able to obtain relative recapture rates in the presence of multiple marks, since we do not observe any mark type with $100 \%$ probability. To incorporate the complexity of the different mark types used, we consider models analogous to those described in Sect. 3.1, but allowing for the recapture probability to differ between the different mark types. In the most general case, allowing for time-dependence, we can reparametrise the model in the form:

$$
\begin{aligned}
p_{\text {tag }}(t) & =h_{\text {tag }}(t) p_{\text {brand }}(t) ; \\
p_{\text {pelage }}(t) & =h_{\text {pelage }}(t) p_{\text {brand }}(t) .
\end{aligned}
$$

The terms $h_{\text {tag }}(t)$ and $h_{\text {pelage }}(t)$ denote the relative capture efficiencies for tagged and pelaged individuals, compared to branded individuals for times $t=2, \ldots, T$. Although we expect branded individuals to be most easily observed, it is not necessary to assume this within the study, and so only place the restrictions that $h_{\text {tag }}(t)$ and $h_{\text {pelage }}(t)$ are non-negative.

Assuming that the field protocol remains consistent over time (from records this would appear reasonable), we may expect the relative recapture rates of the different mark types to be constant over time. This model can be represented by specifying $h_{\text {tag }}(t)=h_{\text {tag }}$ and $h_{\text {peleage }}(t)=h_{\text {pelage }}$ for all $t=2, \ldots, T$. Again, for the reasons discussed above, we would expect $h_{\text {tag }}$ and $h_{\text {pelage }}$ to both be less than one. It is for this reason that we use the branded seals as the reference mark type, for the natural and direct interpretation of the parameters. In addition, due to the difficulties of matching photographs of pelage markings from different study years, we might expect $h_{\text {pelage }}<h_{\text {tag }}$, but again do not impose any such restrictions in this case. 


\section{Simulation study}

We investigate the impact of the effects of ignoring the recapture heterogeneity on the estimation of the survival probabilities via a simulation study. We consider the two issues of mark-loss and recapture efficiency, separately. For all simulated data sets described below, we assume that the survival probabilities are time dependent, and condition on the number of marks applied each year for each mark type as given in Table 1, to replicate similar data sets to the observed data. We provide a brief summary of the results of the simulation study for the recapture efficiency and mark-loss models.

\subsection{Recapture efficiency}

To assess the impact of the mark-dependent recapture probabilities we simulate capture-recapture data from the model $p_{m} / \phi(t)$, using four different combinations of recapture probabilities (or relative recapture efficiency rates) over the different mark types. We essentially consider combinations of relatively high and low recapture probabilities for tagged and pelaged animals, assuming a consistently high recapture probability for branded animals. We estimate the survival probabilities for the two models: $p / \phi(t)$ (constant recapture probability) and $p_{m} / \phi(t)$ (mark-dependent recapture probabilities) with both models having time-dependent survival probabilities. We estimate the survival probabilities for the two models fitted to the simulated data and find (as expected) a negative bias for estimates of the survival probabilities when ignoring the mark dependence of the recapture probabilities.

\subsection{Mark-loss}

To assess the impact of mark-loss we simulate capture-recapture data from the model $p / \phi(t), a_{t a g}$ for various realistic values of $a_{t a g}$. We fit the true model to the data, and the analogous model ignoring tag loss, $p / \phi(t)$. Once more, as expected, a negative bias is observed for the estimates of the survival probabilities for the model $p / \phi(t)$, with the magnitude of the bias increasing with increasing tag-loss rates.

Further details of the simulation study with tabulated results comparing the different estimates obtained from the models are given in the "Appendix".

\section{Results}

A number of models are fitted to the grey seal data, each one representing a competing biological hypothesis. They are compared using Akaike's Information Criterion (AIC; Akaike 1974). Due to the number of possible models that could be considered (i.e. time dependent, mark dependent, mark-loss models and recapture efficiency models) a two-step search algorithm is implemented. In the first-step we consider all possible models with arbitrary time and mark dependence in the survival and recapture 
Table 2 The different models fitted to the data in increasing order of $\Delta$ AIC values for (a) standard mark/time dependent models and (b) mark-loss and recapture-efficiency models, providing the $\Delta \mathrm{AIC}$ values, number of estimable parameters within the model, denoted by $n$, and AIC weights denoted by $w$

\begin{tabular}{llll}
\hline Model & $n$ & $\Delta \mathrm{AIC}$ & $w$ \\
\hline
\end{tabular}

(a) Standard time/mark dependent models

\begin{tabular}{|c|c|c|c|}
\hline$* p_{m} / \phi(t)$ & 21 & 0.0 & 0.97 \\
\hline$p_{m}(t) / \phi(t)$ & 64 & 7.3 & 0.03 \\
\hline$p_{m}(t) / \phi_{m}(t)$ & 91 & 30.6 & 0.00 \\
\hline$p_{m} / \phi_{m}(t)$ & 50 & 35.7 & 0.00 \\
\hline$p_{m}(t) / \phi_{m}$ & 50 & 53.4 & 0.00 \\
\hline$p_{m} / \phi_{m}$ & 6 & 62.5 & 0.00 \\
\hline$p_{m}(t) / \phi$ & 48 & 64.4 & 0.00 \\
\hline$p_{m} / \phi$ & 4 & 74.8 & 0.00 \\
\hline$p(t) / \phi(t)$ & 35 & 76.2 & 0.00 \\
\hline$p / \phi(t)$ & 19 & 79.5 & 0.00 \\
\hline$p(t) / \phi_{m}(t)$ & 64 & 98.5 & 0.00 \\
\hline$p / \phi_{m}(t)$ & 48 & 103.9 & 0.00 \\
\hline$p(t) / \phi_{m}$ & 21 & 122.0 & 0.00 \\
\hline$p(t) / \phi$ & 19 & 134.5 & 0.00 \\
\hline$p / \phi_{m}$ & 4 & 145.1 & 0.00 \\
\hline$p / \phi$ & 2 & 160.0 & 0.00 \\
\hline \multicolumn{4}{|c|}{ (b) Mark-loss and recapture-efficiency models } \\
\hline$p(t), h_{\text {tag }}, h_{\text {pelage }} / \phi(t)$ & 37 & 0.0 & 0.35 \\
\hline$p(t), h_{\text {tag }}, h_{\text {pelage }} / \phi(t), a_{\text {tag }}$ & 38 & 2.0 & 0.13 \\
\hline$p(t), h_{\text {tag }}, h_{\text {pelage }} / \phi(t), a_{\text {pelage }}$ & 38 & 2.0 & 0.13 \\
\hline$* p_{m} / \phi(t)$ & 21 & 2.3 & 0.11 \\
\hline$p_{\text {brand }}(t), h_{\text {pelage }}, p_{\text {tag }}(t) / \phi(t)$ & 54 & 2.9 & 0.08 \\
\hline$p_{\text {brand }}(t), p_{\text {pelage }}(t), h_{\text {tag }} / \phi(t)$ & 47 & 3.5 & 0.06 \\
\hline$p(t), h_{\text {tag }}, h_{\text {pelage }} / \phi(t), a_{\text {tag }}, a_{\text {pelage }}$ & 39 & 4.0 & 0.05 \\
\hline$p_{m} / \phi(t), a_{t a g}$ & 22 & 4.3 & 0.04 \\
\hline$p_{m} / \phi(t), a_{\text {pelage }}$ & 22 & 4.3 & 0.04 \\
\hline$p_{m} / \phi(t), a_{\text {tag }}, a_{\text {pelage }}$ & 23 & 6.3 & 0.02 \\
\hline$p_{m}(t) / \phi(t)$ & 64 & 9.6 & 0.00 \\
\hline
\end{tabular}

Note that the model $p_{m} / \phi(t)$ is common to (a) and (b) and marked $*$ for ease of comparison between the tables, but the $\triangle \mathrm{AIC}$ values and AIC weights are recalculated for each individual table

parameters - a total of $4^{2}=16$ possible models. The AIC statistic, corresponding AIC weight and number of (estimable) parameters of each model is given in Table 2a.

Typically, we interpret models with a $\triangle$ AIC $\leq 2$ as virtually indistinguishable from each other (Buckland et al. 1997). Using this criteria, we identify a single model within this analysis (i.e. model $p_{m} / \phi(t)$ ). However, consider further the model $p_{m}(t) / \phi(t)$ (with $\Delta \mathrm{AIC}$ of 7.3 ). This is a neighbouring model of the optimal model, with a further time-dependence on the recapture probabilities and resulting in an 
additional 33 parameters (due to the different numbers of years for which we have data for the different mark types). The deviance (or log-likelihood) function evaluated at the MLE's of the parameters for model $p_{m}(t) / \phi(t)$ is much greater than that for $p_{m} / \phi(t)$. In the AIC statistic, this increase is penalised by the significantly larger number of parameters in this model to favour the simpler model. However, the recapture-efficiency models suggested in Sect. 3.2 allow a time-dependent recapture probability with a much smaller number of additional parameters, assuming that the relative recapture rates between mark types remains consistent over time. Thus, within the second step of the search algorithm, we consider mark-loss models and recaptureefficiency models, conditional on the given mark and time-dependence identified in these two models.

We begin with considering the relative recapture rate models. For the constant recapture probability model, specifying the model with relative recapture rates is a simple reparameterisation, where $p_{\text {tag }}=h_{\text {tag }} \times p_{\text {brand }}$ and $p_{\text {pelage }}=h_{\text {pelage }} \times p_{\text {brand }}$. Alternatively, for the time-dependent recapture model, specifying the corresponding relative recapture efficiency models reduces the number of model parameters, but still allows a temporal component in the recapture rates. The compromise here is that we assume that the relative recapture rates do not change over time. Finally, conditional on the relative recapture efficiency model identified, we consider models allowing for tag-loss and pelage-loss. These models are shown in Table $2 \mathrm{~b}$, with the corresponding $\triangle \mathrm{AIC}$ values, AIC weights and number of parameters.

The model deemed optimal via the AIC statistic is $p(t), h_{\text {tag }}, h_{\text {pelage }} / \phi(t)$ and does identify a time-dependence on the recapture probabilities (contrary to the case where we only consider arbitrary mark and time dependence) but no mark loss. Models with a $\triangle \mathrm{AIC} \leq 2$ have essentially identical MLE's of the parameters, due to mark-loss rates very close to zero. However, there are a number of models with AIC weights greater than 0.01 (see Table 2b) so we provide model-averaged estimates of the survival probabilities in Fig. 1. The 95\% confidence intervals are calculated using a bootstrap resampling algorithm, stratified by mark type [see Buckland et al. (1997) for further details].

Figure 1 and the models identified in Table 2 both suggest strong temporal dependence on the survival probabilities. There also appears to be evidence for a time-dependent recapture probability, but where the relative recapture probabilities of pelaged and tagged seals to the branded seals are constant over time. In addition, for the different models fitted to the data, tags appear to be the most difficult to re-observe (i.e. have the lowest recapture probabilities). For example, for model $p(t), h_{\text {tag }}, h_{\text {pelage }} / \phi(t)$, deemed optimal by the AIC statistic, the MLE and 95\% confidence interval for $h_{\text {tag }}$ is $0.359(0.255,0.479)$, and for $h_{\text {pelage }}$ is $0.889(0.788,0.945)$. This clearly indicates that, of the marks applied within this study, tags are the most difficult to re-observe. It is estimated that the recapture probability of tagged individuals is significantly lower (between a quarter and a half) than that for branded individuals. In contrast, although there is some difference between the recapture probabilities of branded and pelaged individuals, the difference does not appear to be very great.

We note that for the models considered (in terms of mark and time dependence on the model parameters), adding in tag-loss or pelage-loss essentially provides no improvement in the deviance (or equivalently likelihood) value, evaluated at the MLE 


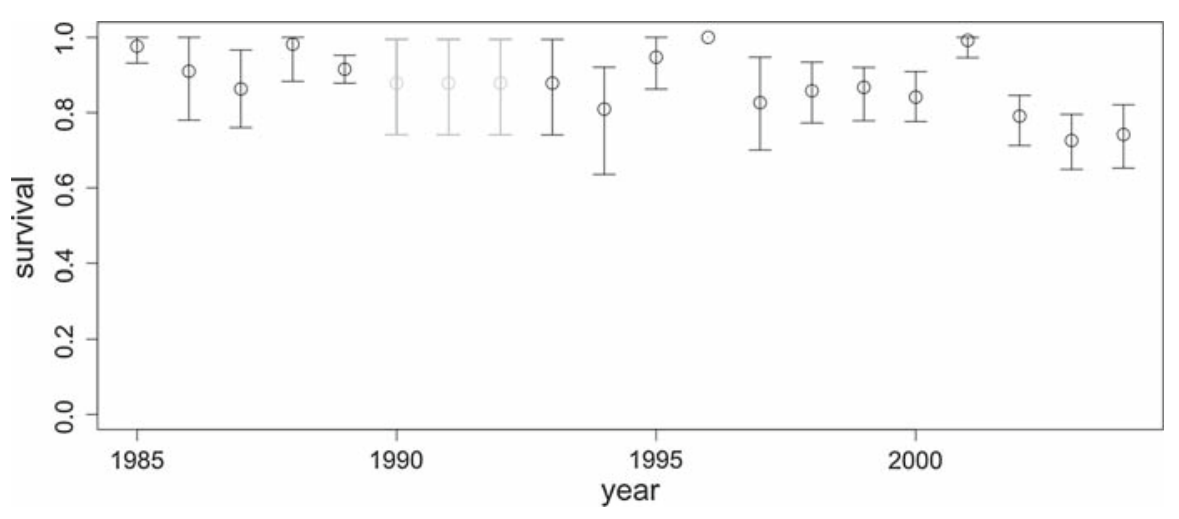

Fig. 1 Model-averaged estimates and 95\% CI for the survival probabilities $\phi_{t}$ based on the models represented in Table $2 \mathrm{~b}$. We note that the years where the survival rate lies on the boundary (i.e. equal to unity) correspond to those where all marked individuals are known to be alive, since they are subsequently observed in later years. The values for 1990-1993 are all set equal to each other since there are no recapture events in the years 1990-1992 so that it is not possible to separately estimate these parameters, only their product. Additionally, we do not present a model-averaged estimate for 2005 since this survival probability is unidentifiable in some of the models (see the discussion on parameter redundancy in Sect. 3)

of the parameters. This is essentially because the estimated tag-loss and pelage loss probabilities are very small. For example, for model $p(t), h_{\text {tag }}, h_{\text {pelage }} / \phi(t), a_{\text {tag }}$ the corresponding MLE (and 95\% CI) for $a_{t a g}$ is $0.003(0.000,0.065)$.

\section{Discussion}

The standard CJS model is commonly used in the modelling of capture-recapture data, and the corresponding survival and recapture parameters estimated. In the presence of multiple mark types applied to different individuals within the same study period, we can consider an integrated analysis, extending the CJS model by essentially considering mark type as an individual covariate. Reparameterising the model allows us to explicitly calculate mark loss probabilities (or relative mark-loss rates) and (relative) recapture efficiency rates. These models are particularly useful when the mark-loss probabilities and/or recapture efficiency terms are constant over time, resulting in both more parsimonious models and removing the problem of parameter redundancy. In addition, ignoring capture heterogeneity in terms of mark dependence typically results in negatively biased estimates of the survival probabilities. The interpretation of the mark-loss probabilities and/or recapture efficiency rates will generally be of interest in any study where different marks can be applied to different animals. There are a number of studies of this kind, for example involving both tagging and DNA-based methods (Meijer et al. 2008), and marking methodology may have animal welfare or conservation implications in some cases (McCarthy and Parris 2004).

Applying the methods to the grey seal data, and using the AIC statistic to compare competing models, we clearly identify the importance of allowing for the different mark types within the analysis. There is strong evidence that the recapture probability 
is dependent on mark type. Branded animals have the highest recapture probability; pelaged animals a slightly reduced recapture probability (approximately 10\% less than branded animals) and tagged individuals the smallest probability of being resighted (approximately only $1 / 3$ of the recapture probability of branded animals). Both branded and pelaged individuals can be identified at a distance of up to $200 \mathrm{~m}$ using either highpowered binoculars or zoom-lensed cameras. However, to observe tagged individuals, the seals need to be seen at close range, at which point tags can still be missed due to being obscured by mud or sand or being covered by the positioning of the seal. From this analysis there is little evidence of pelage loss or tag-loss. However, the number of tagged individuals is small, so that identifying tag-loss is more difficult. Adding tag-loss to the model deemed optimal we obtain a $95 \%$ CI for the tag-loss probability of $(0.000,0.065)$. This interval is relatively wide, and does contain previous estimates of tag-loss for grey seals of around $4 \%$, obtained from other studies where double-tagging was used (Pomeroy et al. 2010).

The models identified within the analysis also strongly indicate a time-dependent survival probability varying between 0.7 and 1 . This is in contrast to other studies on grey seals in different locations. For example, Schwarz and Stobo (2000) obtain survival probability estimates in the range [0.88, 0.92] for young adult seals on Sable Island. The geometric mean of the MLEs of the survival probabilities at NR is 0.882 . This is lower than the time-independent survival estimates of 0.98 for a southern North Sea colony (Gerondeau et al. 2007), and 0.96 (estimated for Norwegian grey seals, Wiig 1991). It appears therefore that the survival probability at NR is generally lower than for grey seals at a number of different locations in several other studies, and may be a large contributing factor in the observed downward trend in pup production at this colony over the time scale of the study (Duck and Mackey 2005). However, in order to fully model colony dynamics at NR, it would be necessary to estimate recruitment into the breeding population and also the levels of permanent emigration and immigration, in addition to adult survival and fecundity.

The underlying causes of the temporal dependence in survival probabilities are unknown. Possible factors include environmental variation, (for example, weather/sea temperatures affecting foraging behaviour or prey availability), anthropogenic factors (for example by-catch in fishing gear or previously undetected culling) and/or density dependent factors. Further work will include the exploration of possible relationships between survival probabilities and external factors. The identification of seals via natural markings is a labour intensive process, but can significantly increase the number of seals in the study using a non-invasive technique. Another area of interest is that some individuals carry more than one mark. Work continues to extend these methods to explicitly model animals that are multiply marked, allowing for both mark-loss probabilities and recapture efficiency rates. In particular, we anticipate that we may obtain an increased precision in the estimates of mark-loss rates using this additional information. Such an approach may also enable us to investigate possible tag-age dependent tag-loss probabilities. For example, "old" tags may have an increased probability of being lost from wear-and-tear; whereas "newly-applied" tags may have an increased probability of being lost from poor application or behaviour of the seal to dislodge the tag. 
Acknowledgments We wish to thank all those involved in the collection of the data at NR, and in particular Sean Twiss, Simon Moss, Paula Redman and Simon Ruddell. We also thank Steve Buckland for discussion of the work within this manuscript and gratefully acknowledge the helpful feedback provided by colleagues at the Sea Mammal Research Unit and Centre for Research into Ecological and Environmental Modelling within the University of St Andrews, especially Len Thomas. We also acknowledge the financial assistance of the UK Engineering and Physical Sciences Research Council in funding the research of Smout, and the UK Natural Environment Research Council in funding ongoing work at North Rona.

\section{Appendix: Simulation study}

To investigate the impact of the effects of ignoring the recapture heterogeneity with respect to the estimation of the survival probabilities we consider a simulation study. In particular, we consider the impact on the estimates of the survival probabilities considering the separate issues of mark-loss and time-invariant recapture efficiency models. We assume that the survival probabilities are time dependent, and are generated from a $U[0.7,0.92]$ distribution. For ease of notation, we let $\phi_{s i m}(t)$ denote the survival probability at time $t$ used for simulating the data. To summarise the estimated bias on the survival probabilities from a simulation study with $N$ simulated datasets, we define the mean percentage error, $\phi_{M P E}$, where,

$$
\phi_{M P E}=\frac{1}{N} \frac{1}{(T-1)} \sum_{i=1}^{N} \sum_{t=1}^{T-1} 100 \times\left(\frac{\hat{\phi}_{i}(t)-\phi_{\text {sim }}(t)}{\phi_{\text {sim }}(t)}\right),
$$

where $\hat{\phi}_{i}(t)$ denotes the MLE of the parameter $\phi(t)$ for dataset $i=1, \ldots, N$. We define the analogous definition for the mean percentage error for recapture probabilities, denoted by $p_{M P E}$. We simulate ten different datasets (i.e. set $N=10$ ) where there are three mark types (brands, tags and pelage), conditioning on the number of marks applied each year of the study for each mark type observed for the UK grey seal data.

\section{Recapture efficiency}

We simulate capture-recapture data from the model $p_{m} / \phi(t)$, conditional on the number of marks applied to individuals at each time period. To assess the impact of the mark-dependent recapture probabilities we consider four possible combinations of recapture probabilities over the different mark types. These are provided in Table 3, along with the corresponding mean percentage error $\phi_{M P E}$, when fitting the correct model $\left(p_{m} / \phi(t)\right)$ and the model that assumes a homogeneous recapture probability, $(p, \phi(t))$. In particular we essentially consider relatively high and low recapture probabilities of tagged and pelaged animals, assuming a consistently high recapture probability for the branded animals.

Clearly, from Table 3 we can see that a relatively low pelage recapture probability results in a larger bias on the estimates for the survival probabilities than a low tag recapture probabilities. This can be explained by the larger number of pelaged animals compared to tagged animals within the study. Once more, ignoring the heterogeneous 
Table 3 The value of the mark-dependent recapture probabilities and corresponding mean percentage error for the estimated survival rates for the models $p / \phi(t)$ and $p_{m} / \phi(t)$

\begin{tabular}{lllll}
\hline pbrand & ptag & $p_{\text {pelage }}$ & \multicolumn{2}{c}{ Mean percentage error, $\phi_{M P E}$} \\
\cline { 3 - 5 } & & & $p / \phi(t)$ & $p_{m} / \phi(t)$ \\
\hline 0.8 & 0.7 & 0.7 & -0.03 & -0.01 \\
0.8 & 0.7 & 0.25 & -3.34 & 0.13 \\
0.8 & 0.25 & 0.7 & -1.14 & -0.41 \\
0.8 & 0.25 & 0.25 & -3.02 & 0.52 \\
\hline
\end{tabular}

Table 4 The tag-loss probabilities and corresponding mean percentage error rates of the estimated survival probabilities for the models $p / \phi(t)$ and $p / \phi(t), a_{\text {tag }}$ fitted to the data

\begin{tabular}{llc}
\hline$a_{\text {tag }}$ & \multicolumn{2}{c}{ Mean percentage error, $\phi_{M P E}$} \\
\cline { 2 - 3 } & $p / \phi(t)$ & $p / \phi(t), a_{\text {tag }}$ \\
\hline 0.01 & -0.10 & 0.27 \\
0.02 & -0.30 & -0.11 \\
0.04 & -0.89 & -0.24 \\
0.08 & -0.93 & -0.14 \\
0.16 & -2.07 & -0.91 \\
\hline
\end{tabular}

recapture probabilities (in terms of mark type) provides a negatively biased estimate of the survival probability. The magnitude of the bias depends on both the difference in the recapture probabilities between mark types (or alternatively relative recapture rates) and also the number of marks applied.

\section{Mark-loss}

We only consider the issue of tag-loss here (and not pelage-loss), since this is thought to be the predominant mark-type for which losses occur. We simulate data from the model $p / \phi(t), a_{t a g}$ for a number of different values of $a_{t a g}$. The values of $a_{t a g}$ considered, and corresponding mean percentage errors for the survival probabilities, $\phi_{M P E}$ when fitting models $p / \phi(t), a_{t a g}$ and $p / \phi(t)$ to the simulated data are provided in Table 4.

The magnitude of the mean percentage error rates for model $p / \phi(t)$ appears to be small (although there is still an apparent negative bias). This is most likely as a result of the relatively small number of tagged animals within the study (only $6 \%$ of the total number of marked animals). This would suggest that it may be difficult to identify adult female tag-loss (if this is small) in the analysis of the real data set. We note that in further simulation studies (not shown here) where a larger proportion of the individuals are regarded as tagged, the negative bias is significantly larger.

\section{References}

Akaike H (1974) A new look at the statistical identification model. IEEE Trans Autom Control 19:716-723 Barrowman NJ, Myers RA (1996) Estimating tag-shedding rates for experiments with multiple tag types. Biometrics 52:1410-1416 
Boyd JM, Laws RM (1962) Observations on the Grey Seal (Halichoerus grypus) at North Rona in 1960. In: Proceedings of the Zoological Society of London, vol 164, pp 469-512

Bradshaw CJA, Barker RJ, Davis LS (2000) Modeling tag loss in New Zealand fur seal pups. J Agric Biol Environ Stat 5:475-485

Buckland ST, Burnham KP, Augustin NH (1997) Model selection: an integral part of inference. Biometrics 53:603-618

Catchpole EA, Morgan BJT, Viallefont A (2002) Solving problems in parameter redundancy using computer algebra. J Appl Stat 29:625-636

Conn PB, Kendall WL, Samuel MD (2004) A general model for the analysis of mark-resight, markrecapture, and band-recovery data under tag loss. Biometrics 60:900-909

Cormack RM (1964) Estimates of survival from the sighting of marked animals. Biometrika 51:429-438

Cowen L, Schwarz CJ (2006) The Jolly?-Seber model with tag loss. Biometrics 62:699-705

Cowen L, Walsh SJ, Schwarz CJ, Cadigan N, Morgan J (2009) Estimating exploitation rates of migrating yellowtail flounder (Limanda Ferruginea) using multistate mark-recapture methods incorporating tag loss and variable reporting rates. Can J Fish Aquat Sci 66:1245-1255

Diefenbach DR, Alt GL (1998) Modeling and evaluation of ear tag loss in black bears. J Wildl Manag 62:1292-1300

Duck CS, Mackey BL (2005) Grey seal pup production in Britain in 2004. Scientific advice on matters related to the management of seal populations: 2005

Gerondeau M, Barbraud C, Ridoux V, Vincent C (2007) Abundance estimate and seasonal patterns of grey seal (Halichoerus grypus) occurence in Brittany, France, as assessed by photo-identification and capture-mark-recapture. J Mar Biol Assoc UK 97:365-372

Hiby L, Lovell P (1990) Computer aided matching of natural markings: a prototype system for grey seals. Technical report, International Whaling Commission, Special issue 12

Jolly GM (1965) Explicit estimates from capture-recapture data with both death and immigration-stochastic model. Biometrika 52:225-247

Kremers WK (1988) Estimation of survival rates from a mark-recapture study with tag loss. Biometrics 44:117-130

Lebreton JD, Burnham KP, Clobert J, Anderson DR (1992) Modeling survival and testing biological hypotheses using marked animals_ a unified approach with case-studies. Ecol Monogr 62:67-118

McCarthy MA, Parris KM (2004) Clarifying the effect of toe clipping on frogs with Bayesian statistics. J Appl Ecol 41:780-786

McMahon CR, White GC (2009) Tag loss probabilities are not independent: assessing and quantifying the assumption of independent tag transition probabilities from direct observations. J Exp Mar Biol Ecol 372:36-42

Meijer T, Noren K, Hellstrom P, Dalen L, Angerbjorn A (2008) Estimating population parameters in a threatened arctic fox population using molecular tracking and traditional field methods. Anim Conserv 11:330-338

Pledger S, Pollock KH, Norris JL (2003) Open capture-recapture models with heterogeneity: I. CormackJolly-Seber model. Biometrics 59:786-794

Pollock K, Hoenig JM, Hearn WS, Callingaert B (2001) Tag reporting rate estimation 1. An evaluation of the high-reward tagging method. N Am J Fish Manag 21:521-532

Pomeroy PP, Fedak MA, Anderson SS, Rothery P (1999) Consequences of maternal size for reproductive expenditure and pupping success of grey seals at North Rona, Scotland. J Anim Ecol 68:235-253

Pomeroy PP, Twiss SD, Duck CD (2000) Expansion of a grey seal (Halichoerus grypus) breeding colony: change in pupping site use at the isle of May, Scotland. J Zool 250:1-12

Pomeroy PP, Smout SC, Twiss SD, Moss S, King R (2010) Low and delayed recruitment at grey seal breeding colonies in the UK (in press)

Reynolds T, King R, Harwood J, Frederiksen M, Wanless S, Harris M (2009) Integrated data analyses in the presence of emigration and tag-loss. J Agric Biol Environ Stat 14:411-431

Schwarz CJ, Stobo WT (2000) Estimation of juvenile survival, adult survival, and age-specific pupping probabilities for the female grey seal (Halichoerus gryprus) on Sable Island from capture-recapture data. Can J Fish Aquat Sci 57:247-253

Seber GAF (1965) A note on the multiple-recapture census. Biometrika 52:249-259

Seber GAF (1982) The estimation of animal abundance and related parameters, 2nd edn. Griffin, London

Testa JW, Rothery P (1992) Effectiveness of various cattle ear tags as markers for Weddell seals. Mar Mamm Sci 8:344-353 
Vincent C, Meynier L, Ridoux V (2001) Photo-identification in grey seals: legibility and stability of natural markings. Mammalia 65:363-372

Wiig A (1991) Demographic parameters for Norwegian grey seals, Halichoerus grypus. Fauna Norvegica Ser A 12:25-28

\section{Author Biographies}

Sophie Smout is a postdoctoral researcher at the University of St Andrews. Her PhD was titled 'Modelling the multispecies functional response of generalist marine predators'. Her research interests include multispecies population modelling and estimating life history parameters from mark recapture data.

Ruth King has been a Lecturer in Statistics at the University of St Andrews since 2003. She completed her PhD at the University of Bristol in 2001 and was a Research Associate at the University of Cambridge from 2001-2003. She also held a UK EPSRC Post-doctoral Fellowship in Mathematics from 2003-2005. Her primary research interests include the analysis of complex capture-recapture data with applications in ecology and epidemiology.

Patrick Pomeroy is a Senior Research Scientist at SMRU, St Andrews University. His research interests include reproductive success in grey seals, variability in life history parameters and behaviour, reproductive energetics and ecotoxicology. 